\title{
Lipid-Rich Plaques Predict Non-Target-Lesion Ischemic Events in Patients Undergoing Percutaneous Coronary Intervention
}

\section{- Insights From Integrated Backscatter Intravascular Ultrasound -}

Tetsuya Amano, MD, PhD; Tatsuaki Matsubara, MD, PhD; Tadayuki Uetani, MD, PhD;

Masataka Kato, MD; Bunichi Kato, MD, PhD; Tomohiro Yoshida, MD; Ken Harada, MD;

Soichiro Kumagai, MD; Ayako Kunimura, MD; Yusaku Shinbo, MD;

Hideki Ishii, MD, PhD; Toyoaki Murohara, MD, PhD

\begin{abstract}
Background: Despite growing interest in non-target lesion events in patients undergoing percutaneous coronary intervention $(\mathrm{PCl})$, there has been little discussion of predictors.
\end{abstract}

\begin{abstract}
Methods and Results: A total of 155 consecutive patients who underwent $\mathrm{PCl}$ were enrolled. Conventional and integrated backscatter intravascular ultrasound (IB-IVUS) parameters were measured in non-target lesions utilizing a 40-MHz intravascular catheter. Lipid-rich plaques (LRP) were defined as lesions with an increased lipid volume (>median) and greater lipid content. Non-target ischemic events were defined as death, non-fatal myocardial infarction, any repeat revascularization and rehospitalization for angina involving the non-target vessel or the target vessel outside the index lesion. During the follow-up period (median: 1,265 days), non-target events were observed in 16 patients (11\%). Using the Cox proportional hazard model, LRP (odds ratio [OR], 6.06; 95\% confidence interval [Cl]: 1.81-20.4, $\mathrm{P}=0.0035)$, elevated serum C-reactive protein (CRP) levels $(\mathrm{OR}, 6.83 ; 95 \% \mathrm{Cl}$ : 2.19-21.3, $\mathrm{P}=0.0009)$ and acute coronary syndrome present at baseline (OR, 4.08; 95\% $\mathrm{Cl}: 1.21-13.8, \mathrm{P}=0.024)$ were significantly and independently associated with non-target events. Synergistic effects of LRP and elevated serum CRP levels for prediction of non-target events (OR, 14.8; 95\% $\mathrm{Cl}$ : 4.57-48.0, $\mathrm{P}<0.0001)$ were found even after adjusting for confounders.
\end{abstract}

Conclusions: LRP measured using IB-IVUS proved to be an independent morphologic predictor of non-target ischemic events after $\mathrm{PCl}$, particularly enhancing the risk in patients with elevated serum CRP levels. (Circ $J$ 2011; 75: 157-166)

Key Words: Integrated backscatter intravascular ultrasound; Non-target-lesion cardiac events; Percutaneous coronary intervention

$\mathbf{P}$ ercutaneous coronary intervention (PCI) using drugeluting stents (DES) has been demonstrated to dramatically reduce re-stenosis and repeat revascularization (RR) inside stented segments..$^{1,2}$ The true impact of PCI, however, can only be fully evaluated against a backdrop of other events resulting from non-target lesions. After successful treatment of the initial culprit lesion by PCI, the risk of a coronary event caused by non-target problems is $5-12 \%$ in the following 1 year and $5 \%$ in each of the subsequent 4 years. ${ }^{3}$ These substantial levels of ongoing morbidity and mortality have led to heightened interest in novel markers to predict coronary events related to non-target lesions. Several plasma markers such as C-reactive protein (CRP) have been advocated for prediction purposes. ${ }^{4}$ Although sampling of peripheral blood to assess risk has the advantages of ease of use and low cost, imaging the actual extent of atherosclerosis in vessels provides improved risk stratification. ${ }^{5}$ Integrated backscatter intravascular ultrasound (IB-IVUS) has recently been developed, allowing analysis of tissue components of coronary plaques in vivo. ${ }^{6-10}$ Using IB-IVUS, we

Received July 4, 2010; revised manuscript received August 24, 2010; accepted September 7, 2010; released online November 23, 2010 Time for primary review: 28 days

Department of Cardiology, Chubu-Rosai Hospital, Nagoya (T.A., T.U., M.K., B.K., T.Y., K.H., S.K., A.K., Y.S.); Department of Internal Medicine, School of Dentistry, Aichi-Gakuin University, Nagoya (T.Matsubara); and Department of Cardiology, Nagoya University Graduate School of Medicine, Nagoya (H.I., T. Murohara), Japan

Mailing address: Tetsuya Amano, MD, PhD, Department of Cardiology, Chubu-Rosai Hospital, 1-10-6 Kohmei, Minato-ku, Nagoya 455-8530, Japan. E-mail: amanot@med.nagoya-u.ac.jp

ISSN-1346-9843 doi:10.1253/circj.CJ-10-0612

All rights are reserved to the Japanese Circulation Society. For permissions, please e-mail: cj@j-circ.or.jp 
sought to determine morphological predictors of non-target cardiac events in patients undergoing PCI, taking into account possible confounding factors.

\section{Methods}

\section{Patients and Study Design}

This study was an observational investigation of 155 consecutive patients who underwent IVUS and PCI for acute coronary syndrome (ACS) and stable angina pectoris between October 2005 and December 2006. ACS included acute myocardial infarction (AMI) and unstable angina pectoris (UAP). The diagnosis of AMI was based on elevation of at least 1 positive biomarker (creatine kinase [CK], CK-MB or troponin $\mathrm{T}$ ), and characteristic electrocardiogram changes and a history of prolonged acute chest pain. UAP was defined as either angina with a progressive crescendo pattern or angina that occurred at rest. Various lipid and inflammatory profiles were measured using commercial radioimmunoassay kits and specific immunoradiometric assays. For this purpose, blood samples were collected from patients before PCI after a 10 12 -h overnight fast. The investigation was approved by the ethics committee of Chubu Rosai Hospital.

\section{Endpoints}

The endpoints were defined as death, non-fatal myocardial infarction, any unplanned repeat revascularization and rehospitalization for angina involving the non-target vessel or the target vessel outside the index lesion. The target lesion was considered to be the area covered by the stents plus $5-\mathrm{mm}$ margins proximal and distal to the edges of the stent. The protocol recommended that multiple interventions for lesions indicated for treatment at baseline should be performed within 30 days after the initial session. Therefore, planned revascularizations were excluded, but not unplanned revascularization or other non-target events within 30 days. Repeat revascularization and rehospitalization were driven by clinical findings (presence of ischemic symptoms with a positive functional ischemia assessment or with an ischemic electrocardiogram change). In order to avoid "oculostenotic" procedures, revascularization for which ischemia was not proved (asymptomatic or negative functional tests) was excluded. The events were assessed by investigators blinded to the IVUS baseline results. Also, each event was confirmed on coronary angiography (CAG) that it was an event relating to segments other than the original target lesion. For multiple occurrences of the same type of event, the time to the first was considered to be the time when an endpoint was reached.

\section{Quantitative CAG and IVUS}

Before performing CAG and PCI, patients were given an intracoronary $0.5-\mathrm{mg}$ dose of isosorbide dinitrate in order to prevent coronary spasm. Quantitative coronary angiography (QCA) was conducted, and the reference diameter and percentage diameter stenosis were measured using a validated automated edge-detection program (CMS-MEDIS Medical Imaging System, Leiden, The Netherlands). Analysis segments ( $\%$ diameter stenosis $<50 \%$ on QCA) in the target and non-target vessels were randomly selected by 2 independent observers unaware of the clinical data. IVUS catheters (40 MHz) were inserted in 155 target vessels and in 49 nontarget vessels as far distally as possible at the index PCI procedure. Continuous ultrasound imaging was acquired during withdrawal of the catheter through the segment of the artery at a constant rate of $0.5 \mathrm{~mm} / \mathrm{s}$. Another coronary artery was analyzed when the selected segments on QCA were $<5 \mathrm{~mm}$ from the target site of PCI and also when the IVUS catheter could not cross the lesion because of severe stenosis. A total of 153 plaques in the target vessels and 52 plaques in the non-target vessels were assessed at baseline. A personal computer (Windows XP Professional, CPU: $3.4 \mathrm{GHz}$ ) equipped with commercially available custom software (IB-IVUS, YD, Nara, Japan) was connected to the IVUS imaging system (Clear View, Boston Scientific, Natick, MA, USA) in order to obtain radio frequency, and signal trigger outputs. Ultrasound backscattered signals were acquired using a $40-\mathrm{MHz}$ (motorized pullback $0.5 \mathrm{~mm} / \mathrm{s}$ ) mechanically rotating IVUS catheter, digitized and subjected to spectral analysis. IB data for each tissue component were calculated as average power levels using a fast Fourier transform, measured in decibels $(\mathrm{dB})$, of the frequency component of backscattered signals from a small volume of tissue. ${ }^{6-9}$

\section{Conventional and IB-IVUS Parameters}

Conventional and IB-IVUS were performed as previously reported. ${ }^{11,12}$ In conventional IVUS, cross-sectional images were quantified for lumen cross-sectional area (LCSA), external elastic membrane (EEM), cross-sectional area (CSA), and plaque $(\mathrm{P})+$ media $(\mathrm{M})$ cross-sectional area $(\mathrm{P}+\mathrm{M}$ CSA $=$ EEM CSA-LCSA) using software built into the IVUS system. An eccentricity index of $\mathrm{P}+\mathrm{M}$ was calculated with the formula of (maximum $\mathrm{P}+\mathrm{M}$ thickness - minimum $\mathrm{P}+\mathrm{M}$ thickness)/maximum $\mathrm{P}+\mathrm{M}$ thickness. The remodeling index was defined as the ratio of EEM CSA at the measured lesion (minimum luminal site) to the reference EEM CSA (average of the proximal and distal reference segments). The eccentricity index and the remodeling index were calculated in the segment with the minimal luminal area. The percentage fibrous area (fibrous area/plaque area, \%FA), the percentage lipid area (lipid area/plaque area, \%LA) and the percentage calcified area (calcified area/plaque area, \%CA) were automatically calculated using the IB-IVUS system. Three-dimensional analysis for conventional and IB-IVUS images was performed to obtain the fibrous volume, lipid volume and calcified volume (separate sums of fibrous, lipid and calcified areas in the segment with the minimum luminal area at 1-mm axial intervals for $5 \mathrm{~mm}$ proximally and for $5 \mathrm{~mm}$ distally). A total of 11 IB-IVUS images were captured at intervals of $1 \mathrm{~mm}$ for a $10-\mathrm{mm}$ length in each plaque. Then, percentages were calculated (fibrous volume/plaque volume, \%FV; lipid volume/plaque volume, \%LV; and calcified volume/plaque volume, $\% \mathrm{CV})$. The variability in $\% \mathrm{LV}$ and $\% \mathrm{FV}$ determined by 2 physicians was also considered from 30 randomly selected records.

\section{Statistical Analysis}

Continuous and categorical variables were assessed as mean \pm SD and percentage, respectively. Univariate analysis was applied to compare QCA and ultrasound parameters between patients with and without non-target ischemic events, using unpaired Student's t-test for continuous normally distributed variables (reference diameter, percentage diameter stenosis, plaque burden, eccentricity index, remodeling index, fibrous volume, percentage plaque volume, $\% \mathrm{LV}, \% \mathrm{FV}$ and $\% \mathrm{CV}$ ), and Mann-Whitney U test for non-normally distributed variables (vessel area, lumen area, plaque area, vessel volume, lumen volume, plaque volume, lipid volume and calcified volume). Chi-squared and Fisher's exact tests were applied when appropriate for categorical variables. The event-free survival rates for clinical events among the groups were ex- 


\begin{tabular}{|c|c|c|c|}
\hline & $\begin{array}{c}\text { Events }(+) \\
\quad(n=16)\end{array}$ & $\begin{array}{c}\text { Events (-) } \\
(n=136)\end{array}$ & $P$ value \\
\hline Male & $11(69)$ & $90(66)$ & $>0.99$ \\
\hline Age (years) & $70 \pm 9.4$ & $69 \pm 9.0$ & 0.61 \\
\hline Body mass index $\left(\mathrm{kg} / \mathrm{m}^{2}\right)$ & $23 \pm 3.3$ & $24 \pm 2.7$ & 0.78 \\
\hline Hypertension (\%) & $11(69)$ & $87(64)$ & 0.79 \\
\hline Diabetes mellitus (\%) & $10(63)$ & $76(56)$ & 0.79 \\
\hline Current smoker (\%) & $5(31)$ & $36(26)$ & 0.77 \\
\hline Old myocardial infarction (\%) & $2(13)$ & $25(18)$ & 0.74 \\
\hline Previous $\mathrm{PCl}(\%)$ & $4(25)$ & $31(23)$ & 0.76 \\
\hline Multiple vessel disease (\%) & $10(63)$ & $70(51)$ & 0.44 \\
\hline Ejection fraction (\%) & $65 \pm 13$ & $66 \pm 12$ & 0.70 \\
\hline Acute coronary syndrome (\%) & $6(38)$ & $24(18)$ & 0.09 \\
\hline LDL-cholesterol (mg/dl) & $119 \pm 33$ & $117 \pm 30$ & 0.85 \\
\hline HDL-cholesterol (mg/dl) & $42 \pm 10$ & $47 \pm 13$ & 0.14 \\
\hline Triglyceride (mg/dl) & $140 \pm 63$ & $145 \pm 91$ & 0.82 \\
\hline CRP (mg/L) & $4.9 \pm 5.1$ & $2.8 \pm 5.0$ & 0.13 \\
\hline ACEI or ARB (\%) & $8(50)$ & $69(51)$ & $>0.99$ \\
\hline$\beta$-blocker (\%) & $8(50)$ & $69(51)$ & $>0.99$ \\
\hline Aspirin (\%) & $15(94)$ & $130(96)$ & 0.55 \\
\hline Ticropidin (\%) & $12(75)$ & $90(70)$ & 0.78 \\
\hline Statins (\%) & $9(56)$ & $79(58)$ & 0.86 \\
\hline Use of DES (\%) & $13(81)$ & $121(89)$ & 0.41 \\
\hline
\end{tabular}

Data are mean \pm SD or no. patients (\%).

$\mathrm{PCl}$, percutaneous coronary intervention; LDL, low-density lipoprotein; HDL, high-density lipoprotein; CRP, C-reactive protein; ACEI, angiotensin-converting enzyme inhibitors; ARB, angiotensin II type 1 receptor blocker; DES, drug-eluting stent.

amined using the Kaplan-Meier method, and the differences in survival rates among the groups were compared using the log-rank test. Cox proportional hazards models were applied to assess univariate and multivariate covariates associated with progression of all patients to clinical events. Lipid-rich plaques (LRP) were defined as increased lipid volume (>median) and greater lipid content $(\% \mathrm{LV}>49 \%$ and $\% \mathrm{FV}<50 \%)$, which occurred in the $75^{\text {th }}$ percentile of $\% \mathrm{LV}$ and $25^{\text {th }}$ percentile of $\% \mathrm{FV}$ in the present study population. ${ }^{11,12}$ The Bland-Altman test was applied to assess concordance of measurements. Multivariate Cox proportional hazards analysis was applied to study the predictors of clinical events adjusting for confounding factors (age, sex and body mass index) and predefined variables. All variables with $\mathrm{P}<0.10$ on univariate analysis were considered in the multivariate model. The time course of changes in the rate of elevated serum CRP levels (>median) between LRP with and without non-target ischemic events was evaluated using 2-way analysis of variance (ANOVA), and Bonferroni test was performed for multiple comparisons. $\mathrm{P}<0.05$ was considered as statistically significant.

\section{Results}

\section{Baseline Patient Characteristics}

Baseline characteristics of patients with or without non-target ischemic events are listed in Table 1. Among 155 patients, 3 were excluded because of low-quality IB-IVUS imaging, leaving a total of 152 patients with 205 mild to moderate lesions (\% diameter stenosis $<50 \%$ ) included in the study. There were no significant differences in baseline data between patients with and without non-target ischemic events. Follow-up was concluded on October 31, 2009. The median follow-up interval was 1,265 days.

\section{Non-Target Events}

During the follow-up period, 16 patients (11\%) developed non-target ischemic events. Each non-target ischemic event was confirmed on CAG to be related to a segment other than that for the original target lesion. Of the 16 non-target events, 7 occurred in plaques outside of the target lesions and the other 9 were attributed to plaques in non-target vessels. There were no deaths. ACS occurred in 6 patients (3 AMI and 3 UAP), and the other events (10 patients) were caused by chest symptom recurrence with a positive functional test or an ischemic electrocardiogram change that led to unplanned catheterization and PCI. A total of 118 patients (78\% of the study population) received routine follow-up angiography. Of those patients who underwent routine follow-up angiography, 18 patients ( $15 \%$ of the study population) underwent revascularization for in-stent re-stenosis. Of the 18 patients (in-stent re-stenosis), 3 patients were found incidentally to have plaque progression in the absence of ischemia, and were then treated with PCI of this non-target lesion. These 3 revascularizations were not included in the study because ischemia was not proven. There were no unplanned revascularizations or adverse events within 30 days.

\section{Interobserver Variability}

The correlation of \% LV and \%FV measured by 2 physicians who conducted IVUS independently was $\mathrm{r}^{2}=0.83(\mathrm{P}<0.001)$ and $\mathrm{r}^{2}=0.79(\mathrm{P}<0.001)$, respectively. In order to validate \% LV measurements by 2 physicians, we compared \%LV measurements on 30 randomly selected lesions. On Bland-Altman plots, the mean differences in $\% \mathrm{LV}$ were $0.22 \pm 8.1 \%$. The limits of agreement for plaque volume were between $-15.7 \%$ and $16.1 \%$. 
Table 2. QCA and IVUS Data vs. Presence of Non-Target Ischemic Events

\begin{tabular}{lccc} 
& $\begin{array}{c}\text { Plaques with events } \\
(\mathbf{n}=\mathbf{8})\end{array}$ & $\begin{array}{c}\text { Plaques without events } \\
(\mathbf{n}=\mathbf{1 9 7})\end{array}$ & P value \\
QCA & & & \\
Reference diameter $(\mathrm{mm})$ & $2.9 \pm 0.3$ & $2.7 \pm 0.6$ & 0.24 \\
Diameter stenosis $(\%)$ & $27 \pm 6$ & $26 \pm 7$ & 0.36 \\
Conventional IVUS & & & \\
Vessel area $\left(\mathrm{mm}^{2}\right)$ & $13.8 \pm 2.8$ & $11.4 \pm 4.2$ & 0.11 \\
Lumen area $\left(\mathrm{mm}^{2}\right)$ & $5.2 \pm 2.5$ & $4.6 \pm 2.2$ & 0.42 \\
Plaque area $\left(\mathrm{mm}^{2}\right)$ & $8.6 \pm 4.4$ & $6.8 \pm 3.3$ & 0.13 \\
Plaque burden $(\%)$ & $63 \pm 14$ & $58 \pm 14$ & 0.36 \\
Eccentricity index & $0.67 \pm 0.23$ & $0.63 \pm 0.20$ & 0.34 \\
Remodeling index & $1.08 \pm 0.15$ & $1.01 \pm 0.18$ & 0.22 \\
Vessel volume $\left(\mathrm{mm}^{3}\right)$ & $160.6 \pm 44.3$ & $123.3 \pm 44.2$ & 0.02 \\
Lumen volume $\left(\mathrm{mm}^{3}\right)$ & $80.6 \pm 43.2$ & $64.4 \pm 26.9$ & 0.10 \\
Plaque volume $\left(\mathrm{mm}^{3}\right)$ & $80.0 \pm 18.5$ & $59.2 \pm 26.9$ & 0.03 \\
$\%$ Plaque volume $(\%)$ & $52 \pm 14$ & $47 \pm 12$ & 0.26 \\
IB-IVus & & & \\
LV (mm $\left.{ }^{3}\right)$ & $41.3 \pm 14.0$ & $22.4 \pm 17.0$ & 0.002 \\
Fibrous volume $\left(\mathrm{mm}^{3}\right)$ & $36.8 \pm 14.4$ & $34.0 \pm 13.9$ & 0.58 \\
Calcified volume $\left(\mathrm{mm}^{3}\right)$ & $1.9 \pm 2.0$ & $2.8 \pm 2.7$ & 0.36 \\
LV $(\%)$ & $52 \pm 17$ & $34 \pm 16$ & 0.004 \\
Fibrous volume $(\%)$ & $46 \pm 15$ & $60 \pm 13$ & 0.003 \\
Calcified volume $(\%)$ & $2 \pm 3$ & $5 \pm 5$ & 0.10 \\
LRP rate $(\%)$ & $5(63)$ & $63(32)$ & 0.029 \\
\hline
\end{tabular}

Data are mean \pm SD or no. plaques $(\%)$.

Data for the percentage diameter stenosis and for cross-sectional area analysis were calculated at the site of minimum luminal area.

IVUS, intravascular ultrasound; QCA, quantitative coronary angiography; IB, integrated backscatter; LV, lipid volume; LRP, lipid-rich plaque.

Table 3. QCA and IVUS Data for Lipid-Rich Plaques vs. Presence of Non-Target Ischemic Events

\begin{tabular}{lccc} 
& $\begin{array}{c}\text { LRP with events } \\
(\mathbf{n = 1 2})\end{array}$ & $\begin{array}{c}\text { LRP without events } \\
(\mathbf{n}=\mathbf{4 8})\end{array}$ & P value \\
QCA & & & \\
Reference diameter $(\mathrm{mm})$ & $3.1 \pm 0.5$ & $3.0 \pm 0.3$ & 0.29 \\
Diameter stenosis $(\%)$ & $33 \pm 11$ & $27 \pm 9$ & 0.07 \\
Conventional IVUS & & & \\
Vessel area $\left(\mathrm{mm}^{2}\right)$ & $16.3 \pm 4.3$ & $14.4 \pm 2.6$ & 0.17 \\
Lumen area $\left(\mathrm{mm}^{2}\right)$ & $5.7 \pm 4.1$ & $5.7 \pm 2.3$ & 0.40 \\
Plaque area $\left(\mathrm{mm}^{2}\right)$ & $10.7 \pm 4.6$ & $8.6 \pm 2.8$ & 0.13 \\
Plaque burden $(\%)$ & $66 \pm 20$ & $60 \pm 14$ & 0.25 \\
Eccentricity index & $0.67 \pm 0.26$ & $0.65 \pm 0.23$ & 0.37 \\
Remodeling index & $1.06 \pm 0.14$ & $1.05 \pm 0.20$ & 0.91 \\
Vessel volume (mm $\left.{ }^{3}\right)$ & $166.7 \pm 47.6$ & $160.6 \pm 30.0$ & 0.58 \\
Lumen volume $\left(\mathrm{mm}^{3}\right)$ & $74.3 \pm 42.4$ & $82.9 \pm 25.0$ & 0.18 \\
Plaque volume $\left(\mathrm{mm}^{3}\right)$ & $92.4 \pm 37.1$ & $77.7 \pm 24.8$ & 0.10 \\
\% Plaque volume $(\%)$ & $57 \pm 17$ & $48 \pm 12$ & 0.05 \\
IB-IVUS & & & 0.09 \\
Lipid volume $\left(\mathrm{mm}^{3}\right)$ & $47.6 \pm 25.1$ & $38.6 \pm 15.2$ & 0.19 \\
Fibrous volume $\left(\mathrm{mm}^{3}\right)$ & $42.8 \pm 16.9$ & $36.8 \pm 13.5$ & 0.49 \\
Calcified volume $\left(\mathrm{mm}^{3}\right)$ & $2.0 \pm 1.7$ & $2.4 \pm 1.9$ & 0.88 \\
Lipid volume $(\%)$ & $51 \pm 13$ & $50 \pm 11$ & 0.98 \\
Fibrous volume $(\%)$ & $47 \pm 12$ & $47 \pm 9$ & 0.27 \\
Calcified volume $(\%)$ & $2 \pm 2$ & $3 \pm 2$ & \\
\hline
\end{tabular}

Data are mean \pm SD or no. patients (\%), and the summation of plaques in a patient. Data for the percentage diameter stenosis and for cross-sectional area analysis were calculated at the site of minimum luminal area.

Abbreviations see in Table 2. 


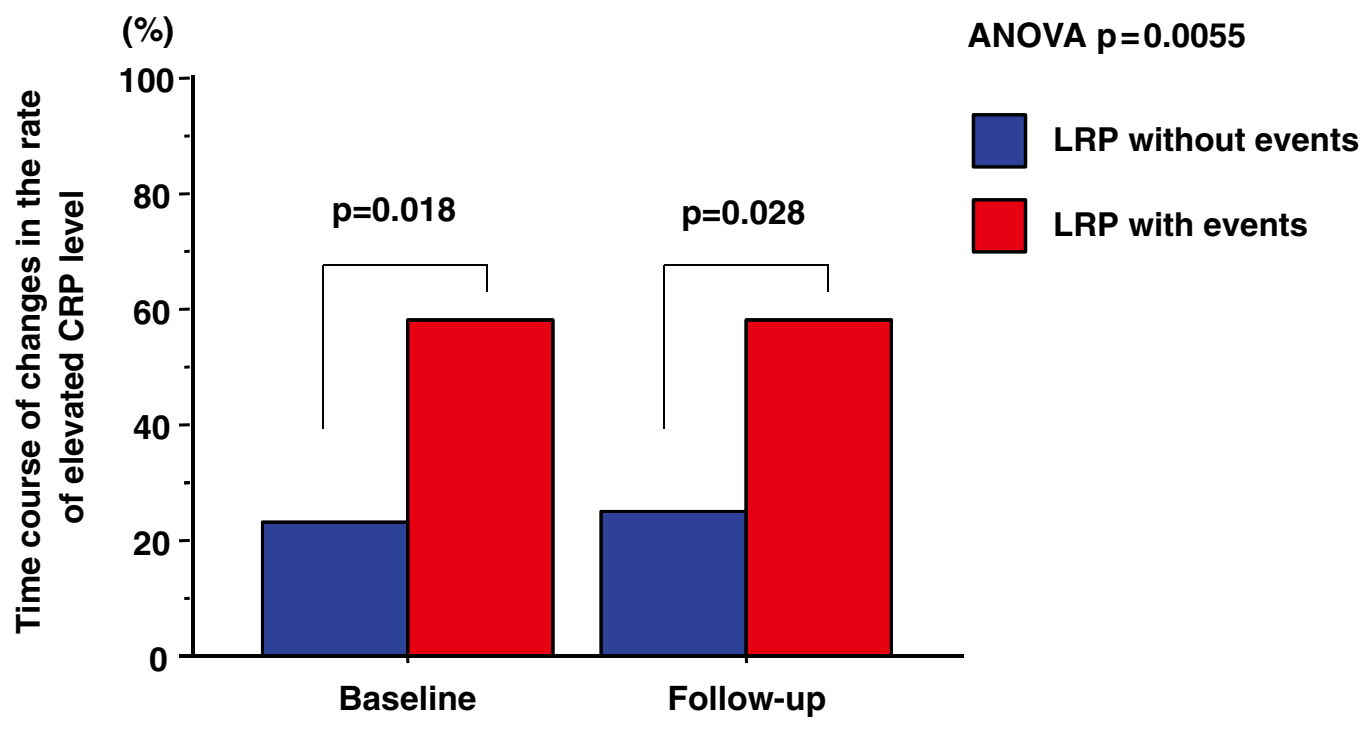

Figure 1. Time course of changes in the rate of elevated serum C-reactive protein (CRP) levels between lipid-rich plaques with or without non-target ischemic events. The rate of elevated serum CRP levels at baseline and at follow-up was significantly greater in the lipid-rich plaques (LRP) with non-target ischemic events compared to LRP without non-target ischemic events (ANOVA $\mathrm{P}=0.0055$ ).

\section{Plaque Analysis for Prediction of Non-Target Events}

Lipid-rich plaque was found in 68 of 205 plaques (33\%). Of the 16 plaques that subsequently caused non-target events, 8 plaques were evaluated on IVUS. Of the 8 plaques that subsequently caused non-target events, $5(63 \%)$ were defined as LRP. IVUS data for the other 8 plaques that led to nontarget events were not available because these 8 plaques had almost normal appearance at baseline or were located in vessels other than those initially assessed on IVUS. In contrast, LRP was found in 63 of 197 plaques unrelated to non-target events (32\%). Table 2 lists the QCA and IVUS data for plaques with or without non-target ischemic events. There were no significant differences in reference diameter (2.9 \pm $0.3 \mathrm{~mm}$ vs. $2.7 \pm 0.6 \mathrm{~mm}$ ) and percentage diameter stenosis ( $27 \pm 6 \%$ vs. $26 \pm 7 \%$ ). On conventional and IB-IVUS analysis, plaques that subsequently caused non-target events (8 plaques) had significantly greater lipid volume with lipid-rich contents as compared to plaques without non-target ischemic events (197 plaques). QCA was performed for all 16 plaques that subsequently caused non-target events at baseline and at follow-up. The reference diameter and \% diameter stenosis at baseline and at follow-up were $2.8 \pm 0.3 \mathrm{~mm}$ and $30 \pm 7 \%$ at baseline, and $2.8 \pm 0.4 \mathrm{~mm}$ and $59 \pm 11 \%$ at follow-up.

\section{QCA and IB-IVUS in LRP Patients}

Of the 152 patients, 60 (39\%) had at least 1 LRP. Table 3 lists the QCA and IVUS data for patients having LRP (60 patients) with non-target ischemic events (12 patients) or patients having LRP without non-target ischemic events (48 patients). The data in Table 3 are from the worst plaque in each patient for each lesion characteristic. There was no significant difference in QCA and IVUS data between the 2 groups except for a tendency for greater plaque burden in patients with non-target ischemic events. As shown in Figure 1, however, the rate of elevated CRP levels (>median, $2.0 \mathrm{mg} / \mathrm{L}$ ) at baseline and at follow up were significantly greater in patients having LPR with non-target ischemic events compared to those without (ANOVA $\mathrm{P}=0.0055$ ).

\section{Kaplan-Meier Analysis}

Figure 2A shows Kaplan-Meier survival curves for nontarget ischemic endpoints according to the presence or absence of LRP. Non-target ischemic events were more frequent in patients with LRP than in those without (log-rank $\mathrm{P}=0.0018$ ). As shown in Figure 2B, patients with elevated serum CRP levels were significantly associated with nontarget ischemic events (log-rank $\mathrm{P}=0.0005)$. Furthermore, when LRP was divided into 2 groups according to the presence or absence of elevated serum CRP levels, LRP with elevated serum CRP levels was significantly and more strongly associated with the increased non-target ischemic events compared to non-LRP and LRP without elevated serum CRP levels (log-rank $\mathrm{P}<0.0001$; Figure 2C).

\section{Cox Proportional Hazard Analysis}

In the univariate Cox proportional hazard model, LRP (odds ratio $[\mathrm{OR}], 5.04 ; 95 \%$ confidence interval [CI]: 1.63-15.6, $\mathrm{P}=0.005)$, elevated serum CRP levels (OR, 5.03; 95\%CI: 1.83-13.8, $\mathrm{P}=0.0018$ ) and LRP with elevated serum CRP levels (OR, 8.99; 95\%CI: 3.34-24.2, P<0.0001) were significantly associated with non-target ischemic events. Greater plaque volume (>median) measured on conventional IVUS and non-LRP with elevated serum CRP levels, however, were not associated with non-target ischemic events (Table 4). In the multivariate model after adjustment for confounders, LRP (OR, 6.06; 95\%CI: 1.81-20.4, $\mathrm{P}=0.0035$ ), ACS present at baseline (OR, 4.08; 95\%CI: $1.21-13.8, \mathrm{P}=0.024)$ and elevated serum CRP levels (OR, 6.83; 95\%CI: 2.19-21.3, P= 0.0009 ) were significantly and independently associated with non-target ischemic events. Furthermore, the presence of LRP and elevated serum CRP levels together (OR, 14.8; 95\% CI: 4.57-48.0, $\mathrm{P}<0.0001)$ provided increased predictive 
A

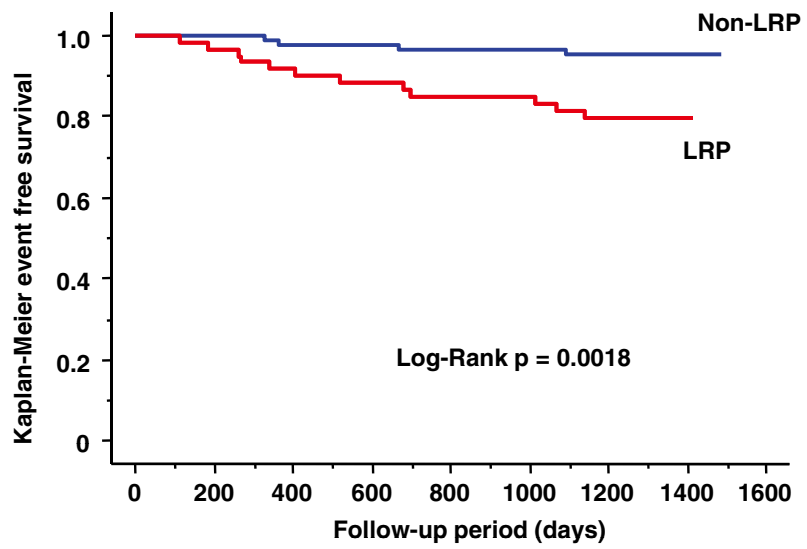

$\begin{array}{lcccc}\text { Number of patients at risk } & 1 \text { year } & 2 \text { year } & 3 \text { year } \\ \text { Non-LRP } & 92 & 90 & 89 & 46 \\ \text { LRP } & 60 & 55 & 54 & 24\end{array}$

B

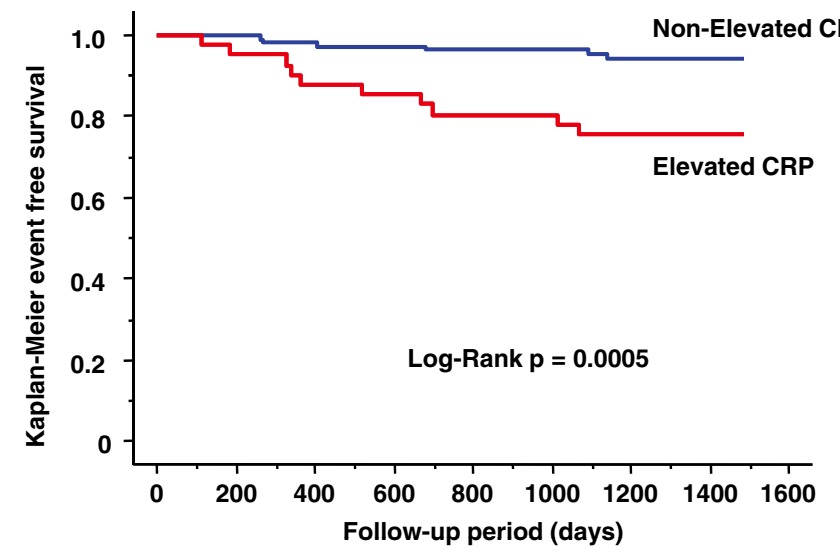

$\begin{array}{lrrrc}\text { Number of patients at risk } & 1 \text { year } & 2 \text { year } & 3 \text { year } \\ \text { Non-Elevated CRP } & 111 & 109 & 107 & 96 \\ \text { Elevated CRP } & 41 & 36 & 33 & 29\end{array}$

C

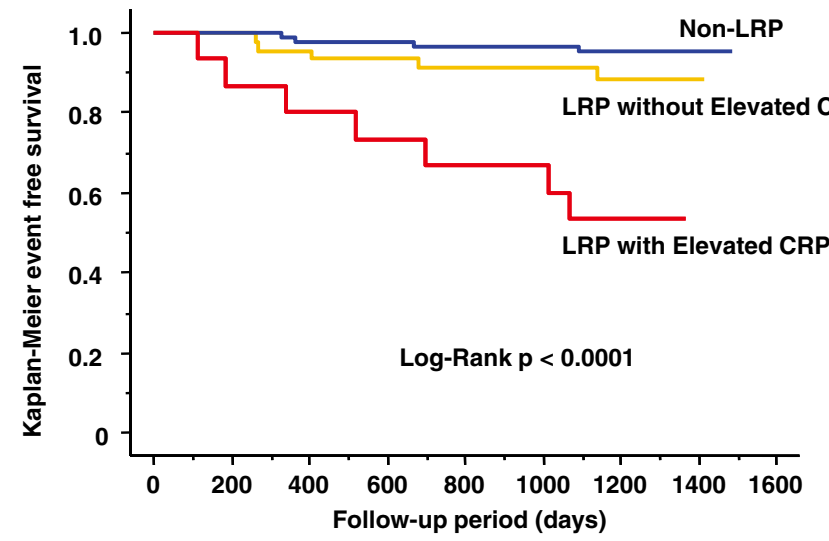

Number of patients at risk

1 year

2 year

3 year

Non-LRP

92

90

89

46

LRP without Elevated CRP 45

43

41

37

LRP with Elevated CRP
Figure 2. Kaplan-Meier estimates: eventfree survival with reference to non-target ischemic events. (A) Non-target ischemic events were significantly higher in patients with lipid-rich plaques (LRP) than in those without (log-rank $\mathrm{P}=0.0018$ ). (B) Elevated serum C-reactive protein (CRP) levels were significantly associated with non-target ischemic events (log-rank $P=0.0005)$. (C) LRP with elevated serum CRP levels (> median) were more strongly associated with non-target ischemic events compared to non-LRP and LRP without elevated serum CRP levels (logrank $\mathrm{P}<0.0001$ ). 


\begin{tabular}{|lcc|}
\hline \multicolumn{2}{|c|}{ Nable 4. Univariate Cox Analysis for Prediction of Non-Culprit Ischemic Events } \\
\cline { 2 - 3 } & \multicolumn{2}{c|}{ Nonsted } \\
Greater plaque volume (> median) & $2.32(0.81-6.67)$ & P value \\
LRP & $5.04(1.63-15.6)$ & 0.12 \\
Elevated serum CRP level (>2 mg/L) & $5.03(1.83-13.8)$ & 0.005 \\
LRP with elevated serum CRP level & $8.99(3.34-24.2)$ & 0.0018 \\
Non-LRP with elevated serum CRP level & $1.33(0.38-4.67)$ & $<.0001$ \\
Acute coronary syndrome & $2.67(0.97-7.36)$ & 0.66 \\
Multiple vessel disease & $1.58(0.57-4.33)$ & 0.057 \\
PCl history & $1.14(0.37-3.54)$ & 0.38 \\
Diabetes mellitus & $1.27(0.46-3.49)$ & 0.82 \\
Hypertension & $1.24(0.43-3.57)$ & 0.64 \\
LDL-cholesterol (mg/dl) & $1.00(0.99-1.02)$ & 0.69 \\
HDL-cholesterol (mg/dl) & $0.96(0.92-1.01)$ & 0.83 \\
Triglyceride (mg/dl) & $1.00(0.99-1.01)$ & 0.13 \\
Smoking & $1.28(0.44-3.68)$ & 0.82 \\
$\beta$-blockers & $0.94(0.35-2.49)$ & 0.65 \\
Statins & $0.93(0.35-2.49)$ & 0.89 \\
ACEl/ARB & $0.99(0.37-2.64)$ & 0.88 \\
\hline
\end{tabular}

$\mathrm{Cl}$, confidence interval; OR, odds ratio. Other abbreviations see in Tables 1,2.

\begin{tabular}{|c|c|c|c|c|}
\hline & \multicolumn{2}{|c|}{ Model 1} & \multicolumn{2}{|c|}{ Model 2} \\
\hline & OR $(95 \% \mathrm{Cl})$ & $P$ value & OR $(95 \% \mathrm{Cl})$ & $P$ value \\
\hline LRP & $6.06(1.81-20.4)$ & 0.0035 & - & \\
\hline Elevated serum CRP level (>2 mg/L) & $6.83(2.19-21.3)$ & 0.0009 & - & \\
\hline LRP with elevated serum CRP level & - & & $14.8(4.57-48.0)$ & $<0.0001$ \\
\hline Acute coronary syndrome & $4.08(1.21-13.8)$ & 0.024 & $4.76(1.36-16.7)$ & 0.015 \\
\hline Age (years) & $1.00(0.93-1.07)$ & 0.95 & $1.01(0.95-1.08)$ & 0.78 \\
\hline Male & $0.97(0.31-2.96)$ & 0.95 & $1.07(0.35-3.27)$ & 0.91 \\
\hline Body mass index $\left(\mathrm{kg} / \mathrm{m}^{2}\right)$ & $1.00(0.84-1.19)$ & 0.98 & $1.02(0.86-1.22)$ & 0.83 \\
\hline
\end{tabular}

Each listed variable (LRP, elevated serum CRP level, LRP with elevated serum level, acute coronary syndrome, age, male and body mass index) was adjusted for all other variables in each model.

Abbreviations see in Tables 1,2,4.

value as compared to LRP and elevated serum CRP levels alone (Table 5). Figure 3 shows images from a patient who subsequently had a non-target ischemic event. This patient had an LRP $(\% \mathrm{LV}=72 \%$ and $\% \mathrm{FV}=26 \%)$ with elevated CRP level $(3.5 \mathrm{mg} / \mathrm{L})$ at baseline.

\section{Discussion}

Although DES implantation has been shown to reduce target cardiac events during year 1 , non-target lesion events dominate during years $2-5$, demonstrating the limitation of PCI as a local therapy. Despite growing interest in non-target lesion events, there has been little discussion of predictors. Non-target lesion events result almost exclusively from progression of disease within other segments of the target vessel or in non-target vessels. ${ }^{3}$ In the present study using IB-IVUS, we found that LRP, not necessarily at non-target event sites, is associated with non-target ischemic events and also significantly and strongly increases this risk in patients with elevated serum CRP levels.

There has been considerable progress in the identification of the molecular and cellular processes causing atherosclerosis, in which the interplay of the accumulation of oxidized low-density lipoprotein in the arterial wall and the actions of macrophages play a critical role. ${ }^{13}$ Histological findings have indicated linkage between larger lipid cores and echolucent (presumably lipid-rich) plaques identified on IVUS. ${ }^{14}$ In the present study many non-target lesion events were caused by RR of an epicardial vessel other than the stented segments, and ACS comprised 6 of 16 events. Although evidence has accumulated that LRP play a critical role in the prevalence of ACS, subclinical plaque rupture of a vulnerable plaque may occur, leading to stepwise plaque progression and worsening of the flow-limiting obstruction by fibrosis and healing, without precipitation of clinically manifest ACS. ${ }^{15-17}$ In some cases, CAG indicated an ACS-like appearance (haziness and thrombus) at follow-up. These patients did not present as having ACS, possibly because of the presence of collateral vessels, although this hypothesis was not directly assessed in the present study. These observations suggest the possibility that LRP are associated with the disease progression as well as with plaque vulnerability.

Recently a study using computed tomography angiography study found a correlation between low-attenuation plaques and acute coronary events. ${ }^{18}$ The findings are difficult to relate to the present study, however, because only a few of the 


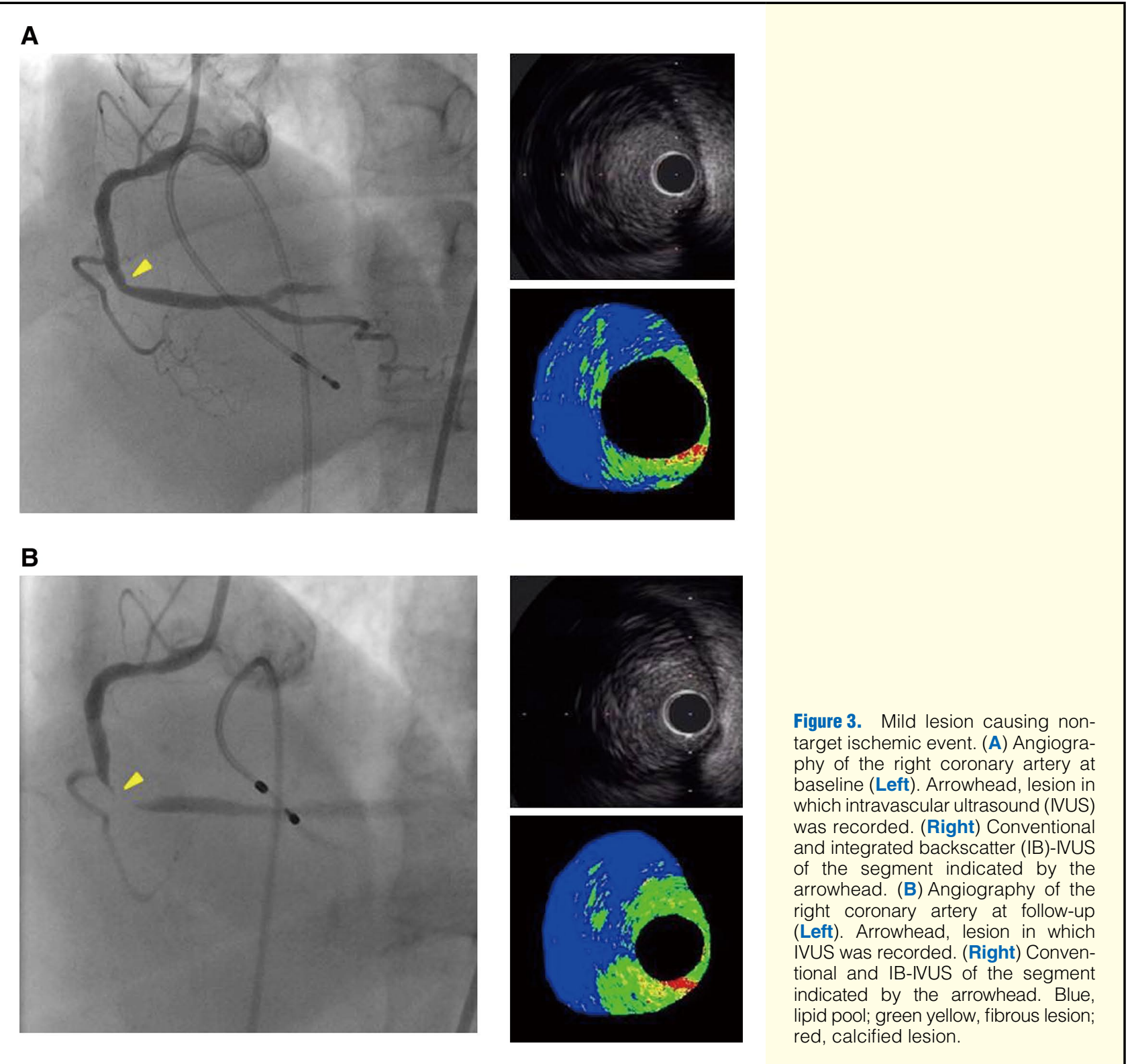

present patients had ACS. A combination of these modalities may provide improved risk stratification with regard to the prediction of future adverse events.

Increased understanding of the processes causing atherosclerosis has facilitated efforts to identify novel markers of risk that may be circulating in plasma. CRP, the most extensively studied plasma marker of inflammation, is 1 such indicator for coronary events, and elevated CRP concentrations have been associated with worse clinical outcomes. ${ }^{19-21}$ Serum CRP level per se has been shown to be related to atherogenesis and atherothrombosis. ${ }^{22-25}$ In the present study, LRP with elevated serum CRP levels had an increased predictive value for non-target lesion ischemic events as compared to LRP and elevated serum CRP levels alone, showing the importance of comorbidity of LRP with inflammation in terms of the activity of an arteriosclerosis pathological change.

Along the same line, another interesting finding was the relationship between ACS present at baseline and future nontarget ischemic events. Studies using angioscopy have sug- gested that, in patients with ACS, all 3 major coronary arteries are widely diseased and have multiple yellow plaques, suggesting the incidence of LRP. ${ }^{26,27}$ LRP closely associated with the instability of the lesions have also been observed in 90$95 \%$ of patients with ACS, in which inflammation of the plaque plays a critical role. ${ }^{28,29}$ These findings have implications regarding the pathophysiology of plaques in patients with ACS, namely the presence of LRP with an aggravation of inflammation in a more diffuse pattern, and are compatible with those of the present study.

The role of statins in the prevention of coronary events is well established, mainly by reducing vascular inflammation and plaque stability. ${ }^{30,31}$ Hence in the present study, the use of statins was not associated with non-target ischemic events, and the non-target events occurred at a comparatively high frequency. This suggests either that most of the included patients in the present study had diabetes, ${ }^{31}$ or that they were not well treated with statins at baseline $(<60 \%)$. From the viewpoint of secondary prevention of coronary events, more 
strict management involving statins is required. Taking these findings together, examinations involving a combination of modalities, such as coronary plaque characterization, biomarkers, and an oral medicine may provide more helpful information in terms of the prediction and prevention of future coronary events.

\section{Study Limitations}

Several limitations need to be considered in the present study. First, the design was single-center and the study population was relatively small. Second, only 8 non-target ischemic events $(50 \%)$ were localized at the lesions initially assessed on IVUS, which precludes any firm conclusion as to the correlation between each plaque composition and risk of adverse outcome. We can conclude, however, that LRP, although not necessarily at the lesion site, may place the patient at risk of a future non-target event. Third, because IB-IVUS does not characterize thin-cup fibroatheroma, it is not clear whether LRP measured on IB-IVUS is a vulnerable plaque predisposing to future acute events. Fourth, thrombus formation is not color coded and analyzed, thereby hindering a rigorous calculation of plaque component in the target lesion, especially in patients with ACS. Hence, in the present study LRP was measured outside of the target lesion, where thrombus formation might be relatively infrequent. We did not detect distinct thrombus formation on gray-scale ultrasound in the present study population. Finally, the observational nature of the present study did not allow us to make definitive conclusions regarding the role of revascularization therapy. The present findings need further confirmation for any preventive or therapeutic implications.

\section{Conclusions}

Lipid-rich plaque measured on IB-IVUS was found to be an independent morphologic predictor of non-target ischemic events after PCI, especially in patients with elevated serum CRP levels. Coronary plaque characterization on IB-IVUS in combination with the assessment of inflammation may offer a novel diagnostic approach to identify patients at risk for future clinical events unrelated to PCI.

\section{Disclosures}

Conflict of interest: There is no conflict of interest for our manuscript. All author: None declared.

\section{References}

1. Moses JW, Leon MB, Popma JJ, Fitzgerald PJ, Holmes DR, O'Shaughnessy C, et al. Sirolimus-eluting stents versus standard stents in patients with stenosis in a native coronary artery. $N$ Engl J Med 2003; 349: 1315-1323.

2. Stone GW, Ellis SG, Cox DA, Hermiller J, O'Shaughnessy C, Mann JT, et al. A polymer-based, paclitaxel-eluting stent in patients with coronary artery disease. N Engl J Med 2004; 350: $221-231$.

3. Cutlip DE, Chhabra AG, Baim DS, Chauhan MS, Marulkar S, Massaro J, et al. Beyond restenosis: Five-year clinical outcomes from second-generation coronary stent trials. Circulation 2004; 110: $1226-1230$.

4. Willerson JT, Ridker PM. Inflammation as a cardiovascular risk factor. Circulation 2004; 109(Suppl): II-2-II-10.

5. Waxman S, Ishibashi F, Muller JE. Detection and treatment of vulnerable plaques and vulnerable patients: Novel approaches to prevention of coronary events. Circulation 2006; 114: 2390-2411.

6. Kawasaki M, Takatsu H, Noda T, Sano K, Ito Y, Hayakawa K, et al. In vivo quantitative tissue characterization of human coronary arterial plaques by use of integrated backscatter intravascular ultrasound and comparison with angioscopic findings. Circulation 2002; 105: $2487-2492$.
7. Sano K, Kawasaki M, Ishihara Y, Okubo M, Tsuchiya K, Nishigaki $\mathrm{K}$, et al. Assessment of vulnerable plaques causing acute coronary syndrome using integrated backscatter intravascular ultrasound. J Am Coll Cardiol 2006; 47: 734-741.

8. Okubo M, Kawasaki M, Ishihara Y, Takeyama U, Yasuda S, Kubota $\mathrm{T}$, et al. Tissue characterization of coronary plaques: Comparison of integrated backscatter intravascular ultrasound with virtual histology intravascular ultrasound. Circ J 2008; 72: 1631-1639.

9. Okubo M, Kawasaki M, Ishihara Y, Takeyama U, Kubota T, Yamaki $\mathrm{T}$, et al. Development of integrated backscatter intravascular ultrasound for tissue characterization of coronary plaques. Ultrasound Med Biol 2008; 34: 655-663.

10. Komura N, Hibi K, Kusama I, Otsuka F, Mitsuhashi T, Endo M, et al. Plaque location in the left anterior descending coronary artery and tissue characteristics in angina pectoris: An integrated backscatter intravascular ultrasound study. Circ J 2010; 74: 142-147.

11. Amano T, Matsubara T, Uetani T, Nanki M, Marui N, Kato M, et al. Impact of metabolic syndrome on tissue characteristics of angiographically mild to moderate coronary lesions: Integrated backscatter intravascular ultrasound study. J Am Coll Cardiol 2007; 49: $1149-1156$.

12. Amano T, Matsubara T, Uetani T, Nanki M, Marui N, Kato M, et al. Abnormal glucose regulation is associated with lipid-rich coronary plaque: Relationship to insulin resistance. JACC Cardiovasc Imaging 2008; 1: 39-45.

13. Libby P, Theroux P. Pathophysiology of coronary artery disease. Circulation 2005; 111: $3481-3488$.

14. Gussenhoven EJ, Essed CE, Lancee CT, Mastik F, Frietman P, van Egmond FC, et al. Arterial wall characteristics determined by intravascular ultrasound imaging: An in vitro study. J Am Coll Cardiol 1989; 14: 947-952.

15. Varnava AM, Mills PG, Davies MJ. Relationship between coronary artery remodeling and plaque vulnerability. Circulation 2002; 105: 939-943.

16. Nishimura RA, Edwards WD, Warnes CA, Reeder GS, Holmes DR Jr, Tajik AJ, et al. Intravascular ultrasound imaging: In vitro validation and pathologic correlation. J Am Coll Cardiol 1990; 16: 145-154.

17. Yamagishi M, Terashima M, Awano K, Kijima M, Nakatani S, Daikoku S, et al. Morphology of vulnerable coronary plaque: Insights from follow-up of patients examined by intravascular ultrasound before an acute coronary syndrome. J Am Coll Cardiol 2000; 35: $106-111$.

18. Motoyama S, Sarai M, Harigaya H, Anno H, Inoue K, Hara T, et al. Computed tomographic angiography characteristics of atherosclerotic plaques subsequently resulting in acute coronary syndrome. J Am Coll Cardiol 2009; 54: 49-57.

19. Servi SD, Mariani M, Mariani G, Mazzone A. C-reactive protein increase in unstable coronary disease. J Am Coll Cardiol 2005; 46: $1496-1502$.

20. Koh KK, Han SH, Quon MJ. Inflammatory markers and the metabolic syndrome. J Am Coll Cardiol 2005; 46: 1978-1985.

21. Ishii H, Toriyama T, Aoyama T, Takahashi H, Amano T, Hayashi $\mathrm{M}$, et al. Prognostic values of C-reactive protein levels on clinical outcome after implantation of sirolimus-eluting stents in patients on hemodialysis. Circ Cardiovasc Interv 2009; 2: 513-518.

22. Devaraj S, Xu DY, Jialal I. C-reactive protein increases plasminogen activator inhibitor-1 expression and activity in human aortic endothelial cells: Implications for the metabolic syndrome and atherothrombosis. Circulation 2003; 107: 398-404.

23. Pepys MB, Hirschfield GM, Tennent GA, Gallimore JR, Kahan MC, Bellotti V, et al. Targeting C-reactive protein for the treatment of cardiovascular disease. Nature 2006; 440: 1217-1221.

24. Nakachi T, Kosuge M, Hibi K, Ebina T, Hashiba K, Mitsuhashi T, et al. C-reactive protein elevation and rapid angiographic progression of nonculprit lesion in patients with non-ST-segment elevation acute coronary syndrome. Circ J 2008; 72: 1953-1959.

25. Matsuo Y, Hashizume T, Shioji S, Akasaka T. Metabolic syndrome is strongly associated with chronic subclinical inflammation in patients achieving optimal low-density lipoprotein-cholesterol levels in secondary prevention of cardiovascular disease. Circ J 2008; 72: 2046-2050.

26. deFeyter PJ, Ozaki Y, Baptista J, Escaned J, Di Mario C, de Jaegere PPT, et al. Ischemia-related lesion characteristics in patients with stable or unstable angina: A study with intracoronary angioscopy and ultrasound. Circulation 1995; 92: 1408-1413.

27. Asakura M, Ueda Y, Yamaguchi O, Adachi T, Hirayama A, Hori M, et al. Extensive development of vulnerable plaques as a pan-coronary process in patients with myocardial infarction: An angioscopic study. J Am Coll Cardiol 2001; 37: 1284-1288. 
28. Hashimoto H, Kitagawa K, Hougaku H, Shimizu Y, Sakaguchi M, Nagai Y, et al. C-reactive protein is an independent predictor of the rate of increase in early carotid atherosclerosis. Circulation 2001; 104: $63-67$.

29. Servi SD, Mariani M, Mariani G, Mazzone A. C-reactive protein increase in unstable coronary disease. J Am Coll Cardiol 2005; 46: $1496-1502$.

30. McMurray JJ, Kjekshus J, Gullestad L, Dunselman P, Hjalmarson A, Wedel $\mathrm{H}$, et al. Effects of statin therapy according to plasma high-sensitivity C-reactive protein concentration in the Controlled
Rosuvastatin Multinational Trial in Heart Failure (CORONA): A retrospective analysis. Circulation 2010; 121: e461.

31. Hiro T, Kimura T, Morimoto T, Miyauchi K, Nakagawa Y, Yamagishi M, et al. Diabetes mellitus is a major negative determinant of coronary plaque regression during statin therapy in patients with acute coronary syndrome: Serial intravascular ultrasound observations from the Japan Assessment of Pitavastatin and Atorvastatin in Acute Coronary Syndrome Trial (the JAPAN-ACS Trial). Circ J 2010; 74: 1165-1174. 